\title{
Stand der mechanischen Löseverfahren im Bergbau
}

\author{
Nikolaus A. Sifferlinger \\ Lehrstuhl für Bergbaukunde, Bergtechnik und Bergwirtschaft, Montanuniversität Leoben, Leoben, Österreich
}

Eingegangen 23. Dezember 2021; angenommen 10. Januar 2022; online publiziert 25. Januar 2022

Zusammenfassung: Während zur Gewinnung großer Tonnagen im Erzbergbau die Sprengtechnik optimal genützt werden kann, ist im Streckenvortrieb untertage die mögliche Vortriebsleistung durch die Notwendigkeit, die giftigen Sprenggase auszuwettern, begrenzt.

Weichere Mineralien wie Kohle, Salz oder Kali lassen sich sehr gut mechanisch gewinnen. Für Reef-Erze mit mittlerer Festigkeit, aber hoher Abrasivität sind mechanische Gewinnungssysteme in Erprobung.

Für den Vortrieb in härteren Gesteinen sind die vorhandenen Teilschnittmaschinen mit Rundschaftmeißeln ungeeignet. Vollschnittmaschinen mit Diskenschneidtechnik sind zwar gut geeignet, aber mit ihren großen Wenderadien und dem kreisrunden Streckenprofil zu unflexibel.

Daher sind derzeit von namhaften Maschinenherstellern Teilschnittmaschinen mit Diskenschneidtechnik in Entwicklung und Erprobung im Streckenvortrieb. Dabei werden tägliche Vortriebsleistungen bis $8 \mathrm{~m}$ erreicht, dies entspricht aber erst etwa $50 \%$ der von der Industrie gewünschten Leistung im Hartgestein.

Weitere Forschung und Entwicklung ist daher notwendig. Auch kombinierte mechanische Lösemethoden, bei denen das Gestein im Gefüge vorgeschwächt wird, können einen Lösungsbeitrag liefern.

Schlüsselwörter: Bergbau, Mechanischer Abbau, Hartgestein, Diskenschneidtechnik

\section{State of the Art in Mechanical Extraction in Mining}

Abstract: For the extraction of large quantities in ore mining, drill and blast operation is an optimal solution. But for underground roadway development, the daily advance

Auf Grundlage eines Vortrages gehalten an der TU Clausthal am 3. Februar 2021 aber mit wesentlichen Ergänzungen.

Univ.-Prof. DI. Dr. mont. N. A. Sifferlinger $(\varangle)$

Lehrstuhl für Bergbaukunde, Bergtechnik und Bergwirtschaft,

Montanuniversität Leoben,

Erzherzog-Johann-Str. 3/5

8700 Leoben, Österreich

Nikolaus-august.sifferlinger@unileoben.ac.at rates are limited by the need of venting out the toxic blast fumes.

Soft minerals like coal, salt, or potash are mainly extracted by mechanical cutting. For Platinum Group Metals, mechanical extraction systems are in testing in reefs with medium rock strength but high abrasivity.

For roadway development in hard rock, roadheaders with pick tools have too much pick wear. Full-face tunnel boring machines with disc cutting tools can extract hard rock well, but their turning radius is too big and they produce circular profiles.

Therefore major mining machine manufacturers have developed and tested prototypes of roadheaders with disc cutting technology. So far these machines have achieved daily advance rates of about $8 \mathrm{~m}$, which is only half of what the industry is asking for in roadway development in hard rock.

Further research and development is necessary. This includes combined methods, where the hard rock is weakened before cutting.

Keywords: Mining, Mechanical excavation, Hard rock, Disc cutting technology

\section{Einleitung}

Während die Sprengtechnik zur Gewinnung großer Tonnagen im Erzbergbau optimal genützt werden kann, hat sie im Vortrieb in den zunehmenden Teufen des Untertagebergbaus Nachteile, welche die Geschwindigkeit des Vortriebes limitieren.

Bedingt durch die Notwendigkeit, die giftigen Sprenggase mit der Ventilation abzuführen, können Sprengungen nur durchgeführt werden, wenn der betroffene Bereich evakuiert ist. In größeren Bergwerken ist das meist nur zu Schichtwechsel praktikabel, und das limitiert die Vortriebsgeschwindigkeiten. Auch wird beim Sprengvortrieb das Gebirge mehr geschädigt als im mechanischen Vortrieb.

Allerdings gibt es derzeit noch Grenzen für den mechanischen Vortrieb im Hartgestein. Daher ist für den Bereich des mechanischen Vortriebs in den letzten Jahren sehr viel 
Forschung und Entwicklung im Gange [1, 2], und dieser Artikel bringt kurz den Stand der mechanischen Lösetechnik im Jahre 2022.

\section{Mechanische Gewinnung}

\subsection{Kohle}

In der Kohle hat die mechanische Gewinnung den Sprengbetrieb ersetzt. Die Auffahrung der Strecken oder die Gewinnung im Room-and-Pillar Mining erfolgt durch Teilschnittmaschinen oder Continuous Miner. Auch Bolter Miner kommen zum Einsatz, welche die Funktion des Schneidens, Förderns und Ankersetzens in sich vereinen. Als Schneidwerkzeuge dienen hauptsächlich Rundschaftmeißel, welche den Anforderungen in der Kohle und bei Bedarf auch im Nebengestein gut entsprechen.

Die modernsten Longwall Mining Systeme im Kohlenbergbau laufen in guten Bedingungen bis zu 98\% im Automatik-Betrieb, überwacht aus der Leitzentrale. Ein Walzenschrämlader der Type CAT EL 3000 kann bis zu 5000 t Kohle pro Stunde gewinnen [3].

Es ist anzumerken, dass für einen reibungslosen Longwall-Betrieb die Flözbedingungen gut und ohne größere Störungen sein müssen.

\subsection{Salz}

Wenn Salz nicht als Sole gewonnen wird, geht der Trend weg vom Bohren und Sprengen zur mechanischen Gewinnung. Damit werden Verunreinigungen durch den Sprengstoff und die Zündeinrichtungen vermieden und die Schädigung des umliegenden Gebirges reduziert.

So hat das deutsche Salzbergwerk Heilbronn seit 2006 die Gewinnung auf Continuous Miner umgestellt, auch um die Sprengerschütterungen unter der Stadt zu vermeiden. Die großen Continuous Miner vom Typ Sandvik MB770 sind in der Lage, im Schnitt 100.000t Steinsalz pro Monat abzu- bauen [4]. Dabei ist es wichtig, die Schneidtrommel und den Schneidvorgang so zu optimieren, dass möglichst wenig zu feines Salz gewonnen wird.

Kritisch für die schneidende Gewinnung von Salz sind Einlagerungen von Anhydrit, der durchaus einaxiale Druckfestigkeiten von $100 \mathrm{MPa}$ UCS (Uniaxial Compressive Strength) erreichen kann. Dies ist zwar für die schneidende Gewinnung auch kein Problem. Da aber die Schneidtrommel für Gewinnung des Steinsalz ausgelegt sind, welches üblicherweise einen UCS-Wert von 15 bis $30 \mathrm{MPa}$ besitzt, ist der Werkzeugabstand für das Schneiden von Anhydrit auf einer "Salzschneidtrommel“ zu groß, und es müssen Schutzvorkehrungen gegen die Beschädigung der Schneidtrommel getroffen werden (Veränderung der Schneidgeschwindigkeiten; zusätzliche Meißel auf der Schneidtrommel).

\subsection{Kali}

Im mechanischen Kalibergbau kommen neben Continuous Minern auch sogenannte Miner Borer (Abb. 1) zum Einsatz. Diese besitzen zwei Schneidräder und in der Sohle und in der Firste je eine Schneidtrommel. In guten Bedingungen schneiden diese Miner Borer je nach Baugröße bis zu 7000 t Kali pro Schicht. Solche Höchstleistungen sind aber nur mit nachgeschalteten kontinuierlichen Fördersystemen erreichbar. Wird diskontinuierlich mit Muldenkippern abgefördert, sinken die möglichen Gewinnungsleistungen wegen der Wartezeiten während der Fahrzeugwechsel.

\subsection{Reef-Erze}

Die Platingruppenelemente (PGE) bilden eine Gruppe von seltenen und edlen Metallen, zu denen Ruthenium, Rhodium, Palladium, Osmium, Iridium und Platin gehören. Diese treten oft in flachen Lagerstätten, dem sogenannten Reef, gemeinsam mit Chromit und Fe-Ni-Cu-Sulfiden auf. Die UCS-Werte für Platinerze im südafrikanischen UG2-Reef
Abb. 1: Sandvik Miner Borer MF 420 Übersichtsbild. Schneidhöhe 3,6 m, Schneidbreite 5,6 m. Installierte Gesamtleistung $1,1 \mathrm{MW}$ bei einem Gewicht von rund $210 t$ Diese Maschine kann in guten Bedingungen bis zu 23 t pro Minute gewinnen (Sandvik)

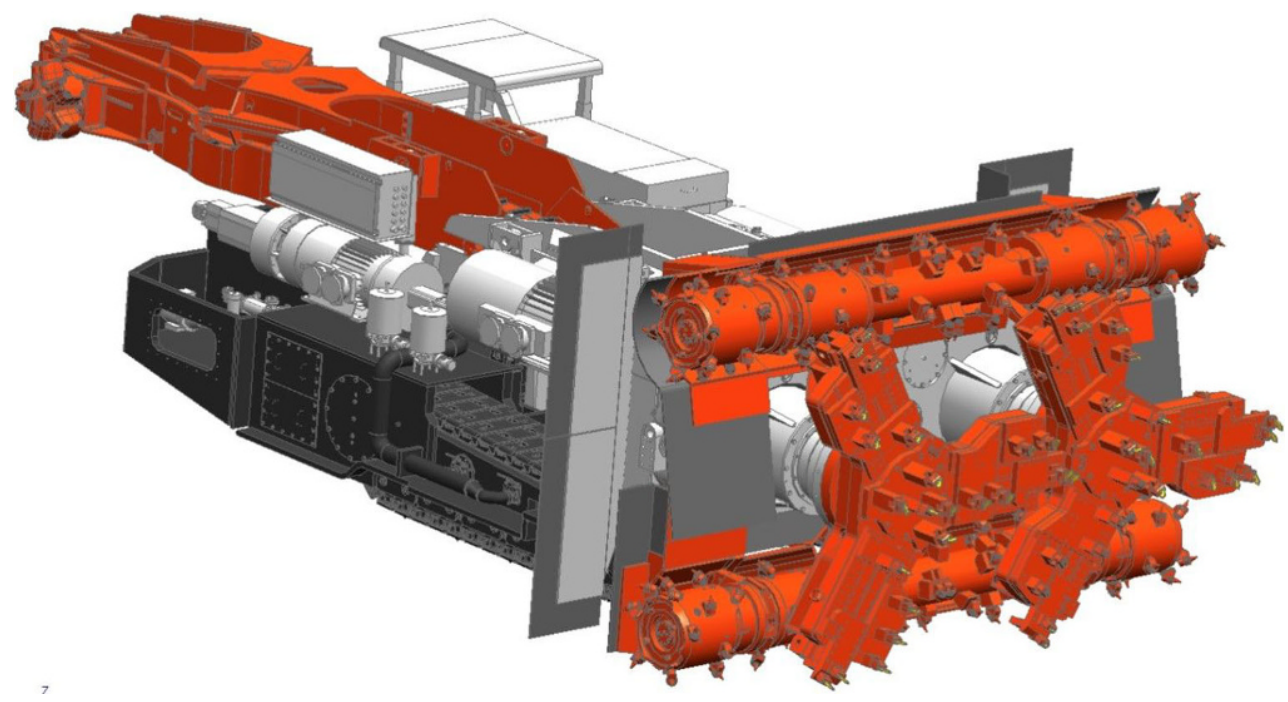




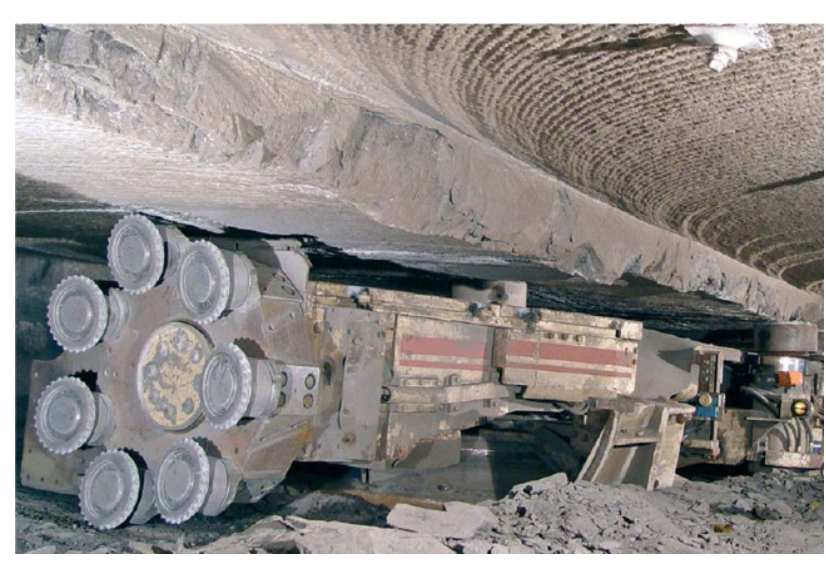

Abb. 2: SandvikReef Miner MN 220 im Probeeinsatz bei Longmin im UG2 Reef(Sandvik)

sind mit bis zu $140 \mathrm{MPa}$ schneidbar, aber die hohe Abrasivität nach Cerchar Abrasivity Index (CAI) von 5,4 ist eine große Herausforderung für die mechanische Gewinnung [5].

Bisher erfolgt die Gewinnung im PGE-Reef sehr personalintensiv durch Bohren und Sprengen, wobei das Reef meist nur eine Abbauhöhe von 1 bis $1,7 \mathrm{~m}$ ermöglicht.

\subsubsection{Reef Miner}

Sandvik hat gemeinsam mit dem Kunden Longmin den Reef Miner MN 220 (Abb. 2) entwickelt. Die Maschine hat die Aufgabe, ferngesteuert eine Länge von $200 \mathrm{~m}$ im Reef aufzufahren, bei einer Schneidhöhe von 1,1 $\mathrm{m}$ und einer maximalen Schneidbreite von 4,3 m. Zu Beginn der Erprobung gab es Probleme mit der Lebensdauer der Schneidwerkzeuge, welche die Strecke von $200 \mathrm{~m}$ ohne Tausch durchhalten müssen. Diese Probleme konnten im Laufe der Erprobungen gelöst werden. Allerdings zeigte sich, dass natürlich diese $32 \mathrm{t}$ schwere Maschine zum Betrieb und Wartung höher qualifiziertes Personal benötigte und dass eine Maschine an die 50 Mann im herkömmlichen Bohr- und Sprengbetrieb ersetzen würde. Daher verliefen die weiteren Einsätze sehr schleppend und der Kunde sah von einer Einführung ab.

Anglo American kündigte 2019 den Einsatz eines Reef Miners MN 220 im Rahmen seines FutureSmart Mining Programmes im südafrikanischen Platinbergbaues im Bergwerk Twickenham an. Ziel sind dabei $3,9 \mathrm{~m}$ Fortschritt pro Schicht und $1200 \mathrm{~m}^{3}$ Erz pro Monat. Sandvik gab im November 2020 bekannt, dass gemeinsam mit Anglo Platinum ein neuer Reef Miner MN 330 in Entwicklung sei. Wenn sich die Nomenklatur der Maschinenbezeichnung nicht geändert hat, wäre das ein System für 1,65 m Schneidhöhe. Der Prototyp findet sich 2021 in der Fertigung und Erprobung im Werk Zeltweg.

\subsubsection{CAT System}

Beim Prototyp des Rock-Straight-System von Cat (Abb. 3) handelt es sich um ein voll mechanisiertes Strebbausystem zum kontinuierlichen Abbau von flachen und platten-

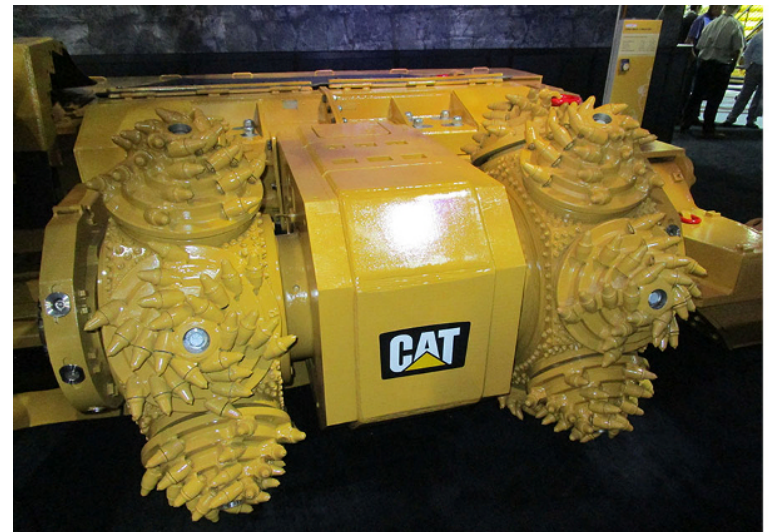

Abb. 3: CAT Rock Straight System Walzenlader HRM220 mit zwei Schneidsystemen mit je $132 \mathrm{~kW}$ Antriebsleistung auf der Las Vegas Mining Show 2016 (Sifferlinger)

förmigen Hartgestein-Lagerstätten wie Platin oder Kupfer. Es kommt ein Hartgestein-Walzenlader zum Einsatz, der mit der speziell für den Abbau von geschichteten Erz-Lagerstätten entwickelten Aktiv-Unterschrämtechnik von Cat arbeitet. Der Hartgesteins-Walzenlader HRM220 ist laut Hersteller für Gesteinsfestigkeiten bis zu $120 \mathrm{MPa}$ UCS ausgelegt. Ein flacher Außenkettenförderer für Hartgestein und hydraulische Hartgestein-Schildausbauten komplettieren das System, das gleichzeitiges Schneiden, Laden und Abfördern, sowie Gebirgssicherung bietet.

Das Rock-Straight-System soll bei Reefen geringer Mächtigkeit von 1,3 bis 2,0 m zum Einsatz kommen. Das System wurde ab 2012 bei KGHM in dem polnischen Kupferbergwerk Polkowice erprobt, ein verbessertes System folgte 2016. Als zukünftige Einsatzgebiete sieht Caterpillar den sambischen Kupfergürtel, die europäischen Kupferschieferlagerstätten und die Bushveld Lagerstätte [6].

\subsection{Surface Miner}

Im Obertagebergbau lassen sich bei entsprechender Lagerstätte Rohstoffe wie Kohle, Gips, Phosphat, Eisenerz, Bauxit oder Kalkstein auch mit Maschinen mit einer Schneidtrom-

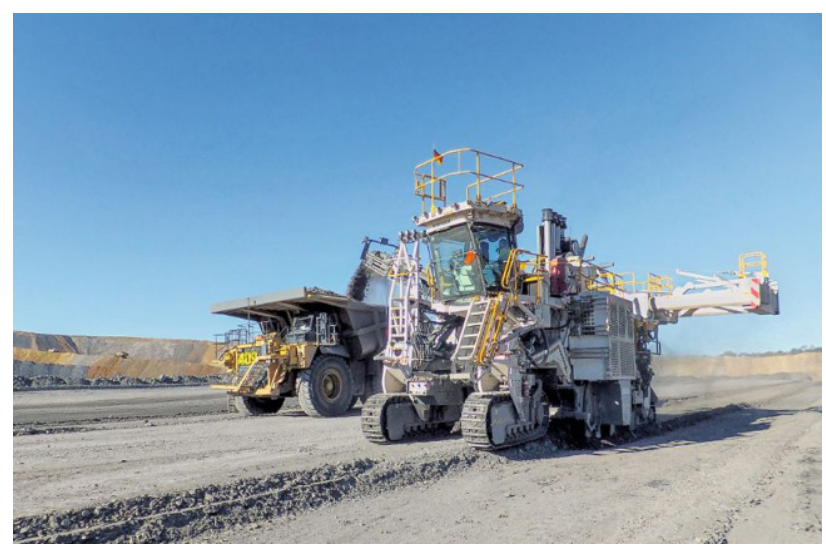

Abb. 4: WIRTGEN Surface Miner schneidet und lädt direkt auf einen Muldenkipper (WIRTGEN) 
Abb. 5: Mechanisches LösenState of the art 2022. Es fällt auf, dass für hohe Gesteinsfestigkeiten und große Flexibilität in Abzweigradien bis $15 \mathrm{~m}$ für mechanisches Lösen derzeit noch eine Lücke herrscht, die es durch Forschung und Entwicklung zu füllen gilt. Die Tunnel Boring Maschinen und Hard Rock Miner sind mit DiskenSchneidtechnologie ausgerüstet, die grundsätzlich sehr hohe Gesteinsfestigkeiten lösen können. Für die Schneidmeißel der Teilschnittmaschinen wird an widerstandfähigeren Materialien geforscht, um auch hier die Grenzen für wirtschaftliches Lösen zu erweitern

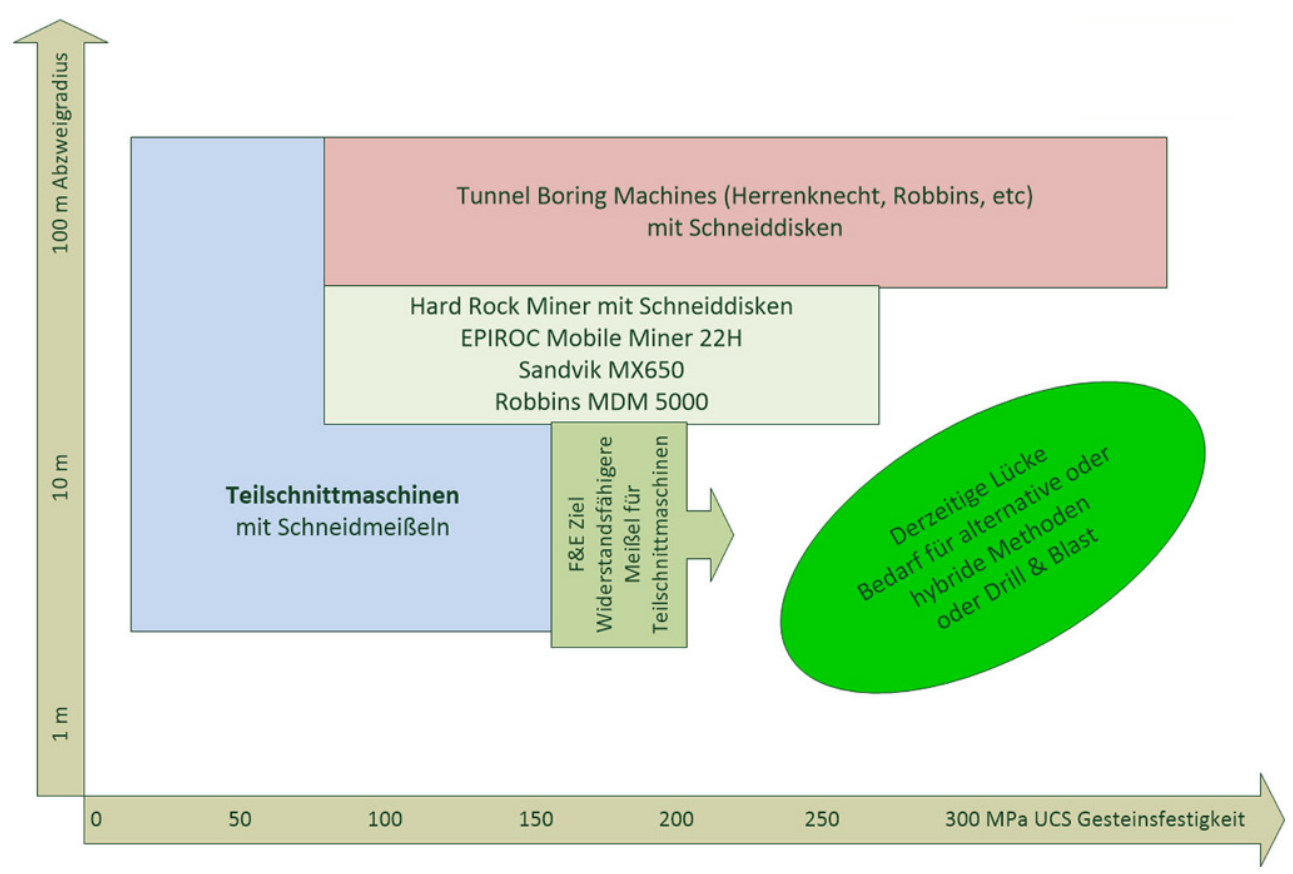

mel gewinnen. Die Firma WIRTGEN ist Weltmarktführer in der Herstellung von Surface Minern (Abb. 4) zur schneidenden Gewinnung im Tagebau. Als Schneidwerkzeuge kommen Rundschaftmeißel zum Einsatz. Der derzeit größte WIRTGEN Surface Miner 4200 SM hat ein Gewicht von $204 \mathrm{t}$, eine maximale Schneidbreite von $4,2 \mathrm{~m}$ und eine maximale Schneidtiefe $0,83 \mathrm{~m}$. Unter optimalen Bedingungen können bis zu $3000 t$ Material pro Stunde gewonnen werden. Die Schneidtrommel ist in ihrer Werkzeugbestückung an die Materialparameter angepasst. Es können Gesteine mit einer einaxialen Druckfestigkeit bis zu $80 \mathrm{MPa}$ wirtschaftlich gelöst werden [7].

Auch für den Surface Miner in der derzeitigen Form ist die Verschleißfestigkeit der Schneidmeißel die Begrenzung gegenüber härteren Gesteinen.

\section{Mechanischer Vortrieb}

Für den mechanischen Vortrieb ist das Forschungs- und Entwicklungsziel derzeit, ein System zu entwickeln, welches die Flexibilität und Beweglichkeit einer Teilschnittmaschine besitzt und dabei Gesteinsfestigkeiten nach UCS bis $300 \mathrm{MPa}$ Iösen kann. Nach Möglichkeit sollte das System auch die Gebirgssicherung nahe der Ortsbrust einbringen können und möglichst automatisch arbeiten.

Dabei soll die Vortriebsleistung im konventionellen Sprengvortrieb von derzeit 8 bis $12 \mathrm{~m}$ pro Tag erreicht und überschritten werden. Die Zielvorstellung für diese Hardrock Mobile Miner liegt bei 15 bis $20 \mathrm{~m}$ Vortrieb pro Tag.

Dabei gibt es aber für Gesteinsfestigkeiten von 150 bis $300 \mathrm{MPa}$ und Abzweigradien $<20 \mathrm{~m}$ immer noch eine "Lücke“ (Abb. 5), für die an alternativen oder hybriden Lösemethoden geforscht wird [1].

\subsection{Teilschnittmaschinen}

Die Teilschnittmaschine mit ihrem beweglichen Schneidkopf, ihrer Mobilität und der Fähigkeit, das geschnittene Material zu laden und fördern, ist für den Streckenvortrieb, der im Bergbau häufig sehr kleine Abzweigradien erfordert, sehr gut geeignet. Eine Teilschnittmaschine kann einen $90^{\circ}$ Abzweig im Streckenvortrieb schneiden, solange die Strecke breit genug ist, um der Teilschnittmaschine das Manövrieren zu ermöglichen (etwa 6 bis $8 \mathrm{~m}$ Streckenbreite).

Die derzeit verfügbaren Schneidmeißel sind jedoch wegen des Verschleißes nicht in der Lage, bei hohen Gesteinsfestigkeiten und hoher Abrasivität wirtschaftlich Material zu lösen. Dabei ist auch die Rock Mass Charakteristik, wie in Abb. 6 gezeigt wird, von Einfluss. Kompaktes Material ist schwerer zu lösen als vorgeschädigtes Material mit

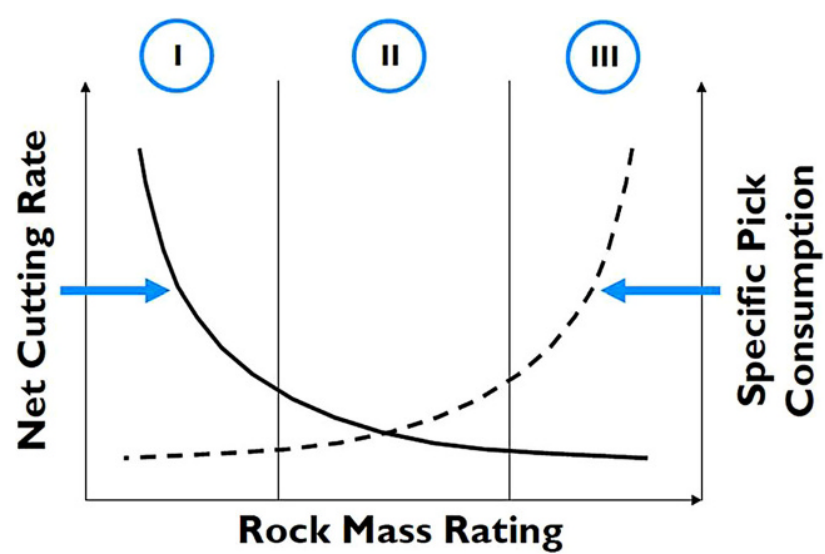

Abb. 6: Der Einfluss der Rock Mass Charakteristik auf die Net Cutting Rate und auf den Spezifischen Meißelverbrauch von Teilschnittmaschinen (Restner und Gehring 2002) 


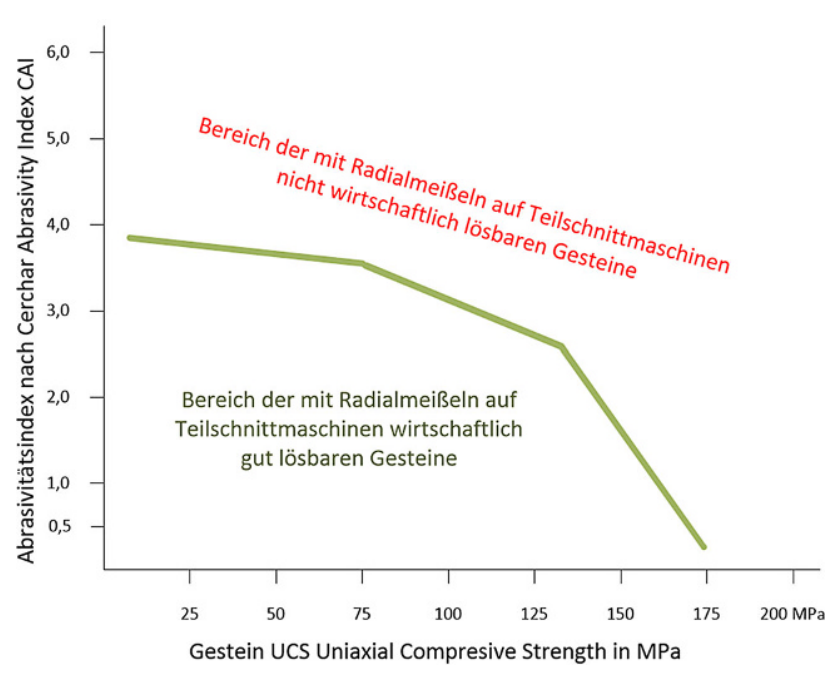

Abb. 7: Abschätzung der wirtschaftlichen mechanischen Schneidbarkeit von Gesteinen in Abhängigkeit von der einaxialen Druckfestigkeit und der Abrasivitätfür die Schneidmeißel von Teilschnittmaschinen. Dabei ist die eingezeichnete Grenze abhängig von den Randbedingungen durchaus keine scharfe, sondern eher ein Bereich, der um etwa $\pm 10 \%$ in beide Richtungen schwanken kann

zahlreichen Klüften [8]. In der Zone I („Reißzone“) wird durch die starke Klüftung der Lösevorgang durch die Aktivierung der Trennflächen sehr stark unterstützt, in der Zone III ( "Schneidzone“) erfolgt der Lösevorgang fast nur mehr durch Schneiden.

\subsubsection{Meißelentwicklung für Teilschnittmaschinen}

Grundsätzlich wären schwere Teilschnittmaschinen, bei Bedarf auch mit Abstützeinrichtungen gegen die Strecke, in der Lage, Gesteinsfestigkeiten nach UCS von bis zu $250 \mathrm{MPa}$ zu lösen. Allerdings sind, wie in Abb. 7 gezeigt, die derzeit verfügbaren Schneidmeißel nicht in der Lage, dies zu leisten.

Die Schneidmeißel mit Wolframcarbid-Spitzen haben trotz intensiver Forschung und Entwicklung in den letzten 20 Jahren nur eine Verbesserung im einstelligen Prozentbereich erfahren.

Kennametal, Sandvik und Element Six haben daher im 21. Jahrhundert massiv in die Entwicklung von Schneidwerkzeugen mit synthetischen polykristallinen Diamanten (PCD) investiert.

Bei den bisherigen Tests haben PCD-Schneidmeißel am Teststand unter stabilen Bedingungen durchaus Gesteine mit einer einaxialen Druckfestigkeit nach UCS von $220 \mathrm{MPa}$ ohne erkennbaren Verschleiß geschnitten. Und dann „zerfallen" sie plötzlich - der genaue Mechanismus ist noch nicht klar - es scheint das Zug- oder Druckkräfte eine Ursache für diese Unstabilität sein könnten.

Noch ist die Lösung für die Instabilität der PCD-Schneidmeißel in höheren Gesteinsfestigkeiten nicht gefunden. Element Six, deren Technologieteil zu 100\% zur De Beers Gruppe gehört, forscht weiter auf diesem Gebiet [9]. Sowohl Element Six als wie auch Kennametal bieten derzeit PCD-Schneidmeißel für den Einsatz in der Kohle und für Straßenfräsen an.
Erst wenn mit der Weiterentwicklung der Schneidmeißel der Durchbruch zu höherer Verschleißfestigkeit gelingt, wird dies den Teilschnittmaschinen neue Einsatzbereiche öffnen. Es ist dies auch die Begründung für die Forschung in alternative oder hybride Lösemethoden, welche in Abschn. 4 kurz angesprochen werden.

\subsection{Tunnel Boring Machines (TBM)}

Tunnel Boring Maschinen (TBM) können mit ihren kreisrunden Schneidrädern, welche mit Schneiddisken besetzt sind, Gestein mit einer einaxialen Druckfestigkeit bis $300 \mathrm{MPa}$ schneiden [10]. Das wäre für Einsätze im Bergbau fast immer ausreichend.

Da aber mit Schneiddisken für die Penetration in das Gestein eine Andruckkraft von bis zu $320 \mathrm{kN}$ pro Schneidwerkzeug erforderlich ist, müssen TBMs diese Kräfte aufbringen können. Daher haben diese Maschinen Gripper, mit denen sie sich abstützen können [11, 12]. Das bedingt große und schwere Maschinen mit mindestens 225t Maschinengewicht für zum Beispiel 4,5 m Schneidraddurchmesser.

Bedingt durch das Schneidrad können diese TBMs nur kreisrunde Profile schneiden und sind im tieferen Untertagebau in Gefahr, durch die Gebirgssetzung eingeklemmt zu werden. Hier ist noch Forschung und Entwicklung im Gange, um die Maschinen besser an diese Bedingungen anzupassen.

Der zweite große Nachteil der TBMs für den Vortrieb im Bergbau ist jedoch der große Abzweigeradius von über $60 \mathrm{~m}$ für die derzeit verfügbaren Systemen $[1,13]$.

Im Bergbau ist häufig das Schneiden von $90^{\circ}$ Abzweigen und ähnlichen Streckenveränderungen notwendig. Daher soll das mechanische Vortriebssystem in der Lage sein, in der Streckenbreite solche Abzweige aufzufahren.

\subsection{Mobile Miner mit Diskenschneidtechnik}

Es gibt Bemühungen, ein Vortriebssystem mit Diskenschneidtechnik und der Flexibilität einer Teilschnittmaschine zu schaffen. Dabei ist zu beachten, dass eine gewisse Mindestanzahl von Disken immer im Schneideingriff sein

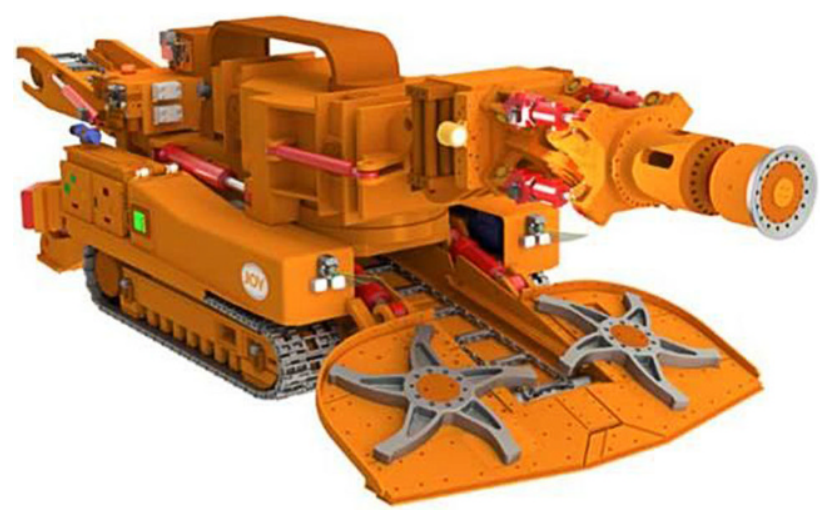

Abb. 8: Der Komatsu DynaMiner mit einer aktivierten Schneiddiske nach dem DynaCut Prinzip (Komatsu Mining) 


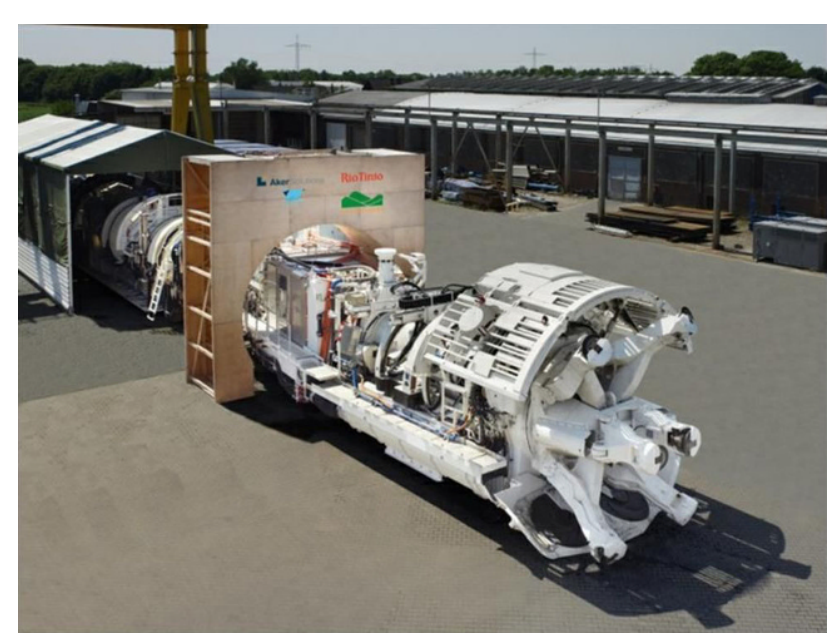

Abb. 9: Aker Wirth Mobile Tunnel Miner MTM 6 Prototyp mit sechs Schneidarmen im Jahre 2012 (Aker Wirth)

muss. um ökonomisch relevante Materialmengen zu lösen. Systeme, die nur eine Diske besitzen, können zwar lösen, aber die Menge ist für den Vortrieb bisher zu gering.

Systeme wie der Komatsu DynaMiner (Abb. 8), der mit dem DynaCut System eine aktivierte Schneiddiske zum Einsatz bringt, dürften aus Sicht des Autors in der Löseleistung zu begrenzt sein. Der DynaMiner Prototyp wurde in der australischen Newcrest Cadia East Mine erprobt [14].

Der Aker Wirth Mobile Tunnel Miner MTM 6 (Abb. 9) war mit sechs Schneidarmen mit je einer Schneiddiske ausgerüstet, und der Prototyp begann im April 2013 im australischen Bergwerk North Parkes seinen Probeinsatz.

Der Verkauf von Aker Wirth im Jahr 2013 an China Railway Technology Equipment scheint alle Aktivitäten mit dem Mobile Tunnel Miner gestoppt zu haben $[1,15]$. Allerdings würde es den Autor nicht überraschen, wenn eines Tages eine chinesische Version des Mobile Tunnel Miner MTM 6 am Markt erscheinen würde.

Es bleibt anzumerken, dass natürlich auch die Vortriebsleistungen von Maschinen mit Diskenschneidtechnik von der Qualität und Lebensdauer der Disken abhängen. Der Tausch einer Schneiddiske ist wegen Größe und Gewicht eine schwere Arbeit. Auch in die Verbesserung der Schneiddisken geht daher entsprechender Forschungs- und Entwicklungsaufwand.

\subsubsection{EPIROC Mobile Miner}

EPIROC als Teil der Atlas Copco Gruppe beschäftigt sich seit den 1970er-Jahren mit dem mechanischen Auffahren von Strecken in Hartgestein. In den 1990er-Jahren war Atlas Copco der Eigentümer von Robbins, und in Australien wurde der Jarva Robbins Mobile Miner mit einem diskenbestückten Schneidrad in Gesteinsfestigkeiten von $300 \mathrm{MPa}$ UCS zum Einsatz gebracht. Die maximale Vortriebsleistung war $80 \mathrm{~m}$ pro Monat. Im Probeeinsatz im Bergwerk Mount Isa in Queensland wurden insgesamt 1,1 km aufgefahren, im Bergwerk Pasminco Broken Hill in New South Wales 1,4 km. Der Autor hatte dabei in Broken Hill die Möglichkeit, die Maschine im Einsatz zu sehen, und hat dabei auch Dick Robbins kennen gelernt. Die Vibrationen auf der Maschine im Schneidbetrieb waren stark, aber erträglich. Ein Problem war die kurze Standzeit der Verschleißteile am Schneidrad und im Fördersystem.

Aufbauend auf diese Erfahrungen hat EPIROC (damals noch Atlas Copco) für Rio Tinto vor 10 Jahren den Mobile Miner 55V entwickelt. In der Bergbaukrise um 2013 hat aber Rio Tinto dann das Interesse verloren, und der bezahlte Prototyp ging zunächst auf Lager. Inzwischen ist er im Probeeinsatz in einer Atommülllager Testgrube in Schweden. ckelt:

EPIROC hat bisher drei Typen des Mobile Miners entwi-

1. Mobile Miner $22 \mathrm{H}$ (das $\mathrm{H}$ steht für horizontal cutting wheel) (Abb. 10 und 11)

- Maschinengewicht 200t

- Maximales Schneidprofil $3 \mathrm{~m}$ Höhe × 5,7 m Breite

- Schneidrad mit 3,5m Durchmesser und 32 Schneiddisken und einer maximalen Andruckkraft von $1800 \mathrm{kN}$
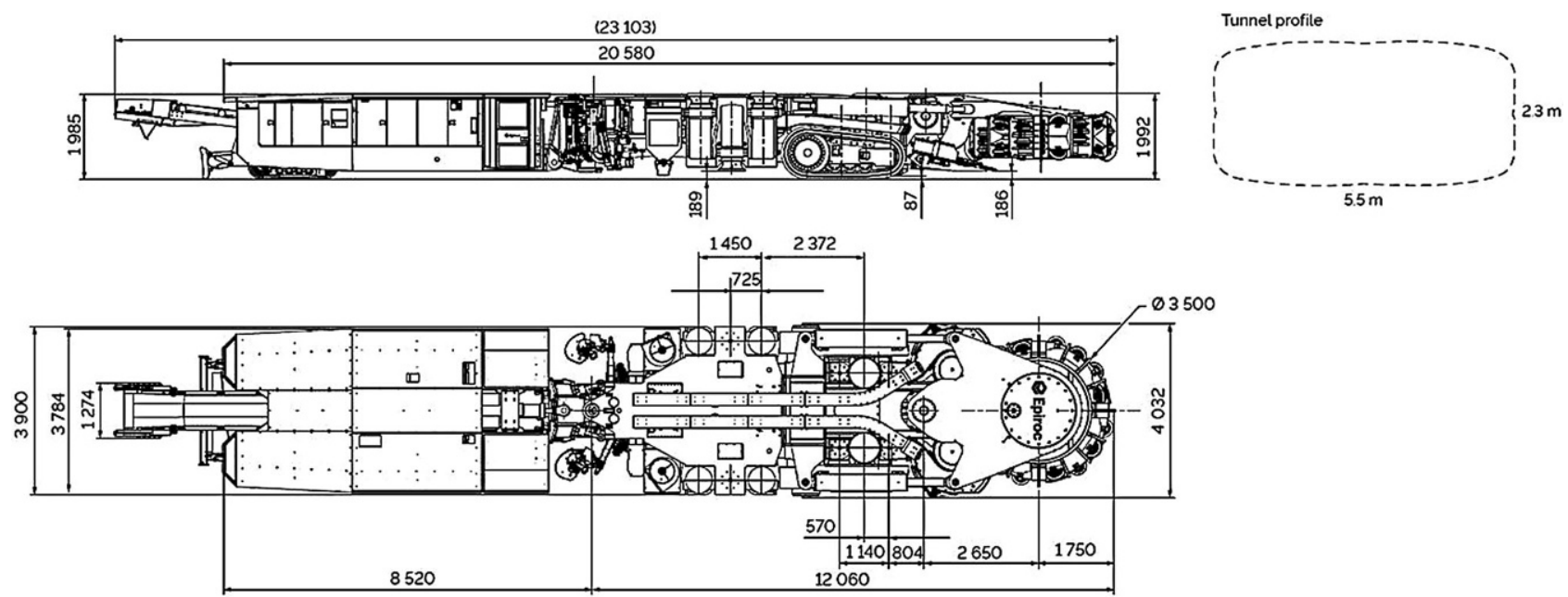

Abb. 10: EPIROC Mobile Miner 22H und das mögliche minimale Schneidprofil von 5,5 m × 2,3m (EPIROC) 


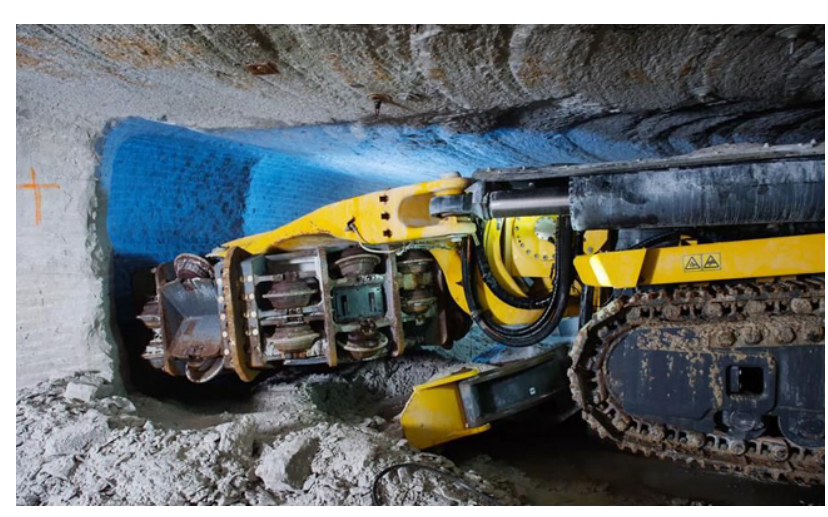

Abb. 11: EPIROC Mobile Miner 22H im Einsatz im südafrikanischen Bergwerk Twickenham (EPIROC)

- Minimaler Abzweigradius $25 \mathrm{~m}$

- Vortriebsleistungen in Abhängigkeit vom Gestein 10 bis $12 \mathrm{~m}$ pro Tag

- Diese Maschine wurde 2016 in Südafrika im Bergwerk Twickenham von Anglo American zum Einsatz gebracht.

2. Mobile Miner 40V

- Schneidprofil $4 \mathrm{~m}$ Höhe $\times 4 \mathrm{~m}$ Breite

- Erwartete Vortriebsleistungen in Abhängigkeit vom Gestein 10 bis $15 \mathrm{~m}$ pro Tag

- Diese Maschine wurde im August 2019 zum Probeeinsatz in das EPIROC Testbergwerk in Kvarntorp in Schweden gebracht.

3. Mobile Miner 55V

- Schneidprofil 5,5 $\mathrm{m}$ Höhe $\times 5,5 \mathrm{~m}$ Breite

Die EPIROC Mobile Miner sind für Gesteinsfestigkeiten von 150 bis $250 \mathrm{MPa}$ UCS ausgelegt. Fernsteuerung und automatischer Schneidzyklus sind verfügbar, im Nachläufer ist auch die Einrichtung zum Ankern zur Gebirgssicherung untergebracht [16-18].

\subsubsection{Sandvik MX650 Hardrock Miner}

Seit den 1990er-Jahren beschäftigt sich Sandvik mit Konzepten für das Rapid Mine Development im Hartgestein.

Im Jahre 2017 wurde der Prototyp des Sandvik MX650 Hartgesteinsminers (Abb. 12) im österreichischen Wolf-

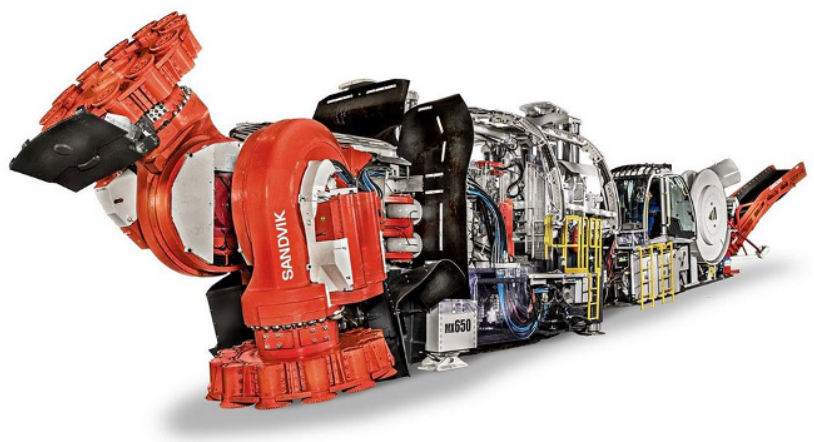

Abb. 12: Der erste Prototyp des Sandvik MX650 vor dem Ersteinsatz in Mittersill im Jahre 2017. Nach den ersten Erfahrungen wurden 2018/2019 zahlreiche Modifikationen durchgeführt (Sandvik)
ram-Bergwerk Mittersill, welches auch zur Sandvik Gruppe gehört, zum Ersteinsatz gebracht. Es stellte sich heraus, dass Ladeeinrichtung, Stabilisierung und Schneidraddetails massiv überarbeitet werden müssen. Nachdem diese Umbauten und Adaptionen erfolgt waren, ging der modifizierte Prototyp im Jahre 2019 in einen weiteren Einsatz. Der Autor konnte die geschnittene Strecke und die in Wartungsschicht befindliche Maschine im Februar 2020 in Mittersill befahren. Die Vortriebsleistung lag bei $4 \mathrm{~m}$ pro Schicht in Gesteinsfestigkeiten bis zu $250 \mathrm{MPa}$ UCS, das Streckenprofil war sehr sauber geschnitten. Die Schneideinrichtung funktionierte gut.

Sandvik hat die Maschine möglichen Kunden im Einsatz vorgeführt, ist aber sonst mit Veröffentlichungen sehr restriktiv.

Nachdem die Funktion des Prototyps praktisch nachgewiesen wurde, wird nun den Aussagen des Präsidenten von Sandvik Mining and Rock Technology, Henrik Ager, im November 2020 nach zu schließen, an einer kommerziellen Version des MX650 Rapid Mine Development Systems gearbeitet. Er gab auch die Entwicklung eines neuen MN330 Narrow Reef Production Systems für Anglo Platinum bekannt $[19,20]$.

Im Jahr 2021 gab es Gespräche mit einem kanadischen Bergbaukonsortium, welches den verbesserten Prototypen noch einmal kurz in Mittersill einsetzen wollte und dann mit den Ergebnissen eine neue Version der Maschine bestellen würde.

\subsubsection{Robbins Mine Development Machine MDM 5000}

Robbins hat mit der Mine Development Machine MDM 5000 eine Vortriebsmaschine für ein rechteckiges Profil von $5,0 \times 4,5 \mathrm{~m}$ mit Diskenschneidtechnik entwickelt ([21, 22]; Abb. 13, 14 und 15).

Bei dieser Maschine werden die linear angeordneten Schneiddisken auf und ab bewegt.

Der Prototyp wurde im mexikanischen Silberbergwerk Fresnillo auf der Ebene $-695 \mathrm{~m}$ erprobt. Dort wurde eine Strecke in Andesit und Schiefer mit Quarzeinlagerungen in Gesteinsfestigkeiten um $200 \mathrm{MPa}$ aufgefahren. Dabei wur-

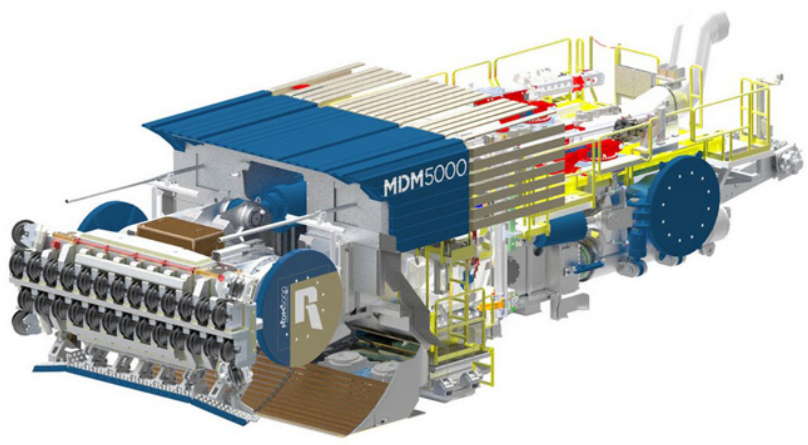

Abb. 13: 3D-CAD Bild des Robbins MDM5000. Man beachte die beiden runden Gripper an jeder Maschinenseite, welche die resultierenden Schneidkräfte zum Teil aufnehmen. Für die Gebirgssicherung befinden sich Ankerbohr- und Setzlafetten an Bord. (The Robbins Company) 


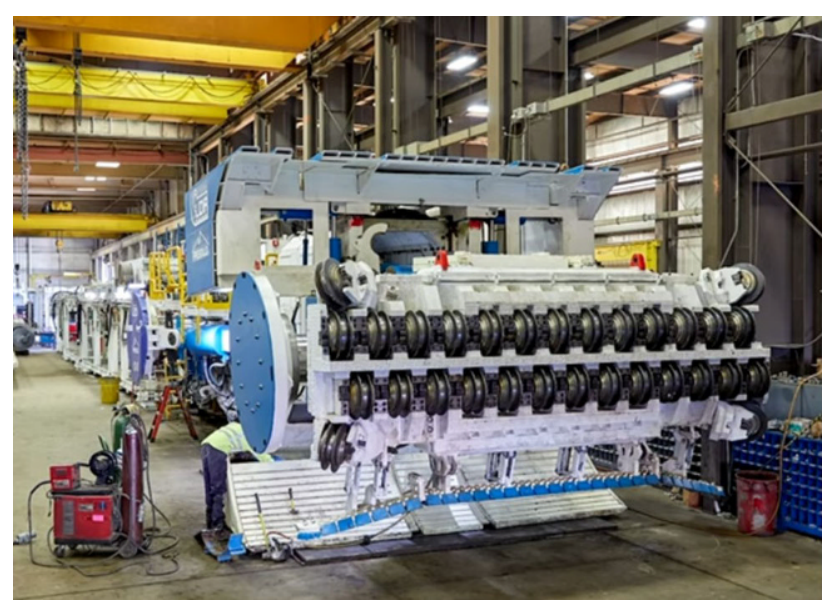

Abb. 14: Robbins MDM5000 Prototyp in der Werkstätte. Man beachte den Nachläufer zur Versorgung der Maschine, welcher den möglichen Kurvenradius sicher einschränkt. Die Diskenschneideinrichtung wird im Einsatz auf und ab bewegt. (The Robbins Company)

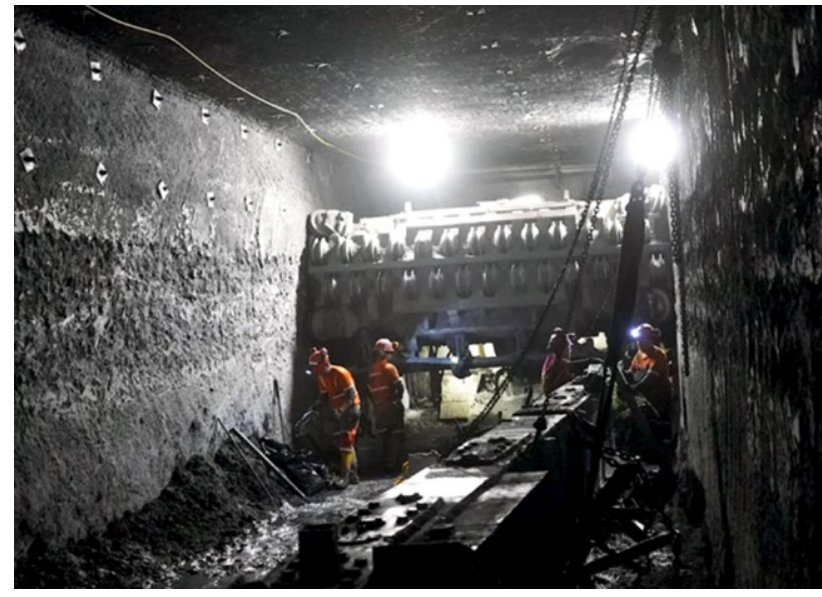

Abb. 15: Robbins MDM 5000 im Bergwerk Fresnillo in Mexiko (The Robbins Company)
Abb. 16: Übersicht über die wesentlichen Gesteinslösemethoden nach dem derzeitigen Stand des Wissens

\section{Rock Extraction Methods}

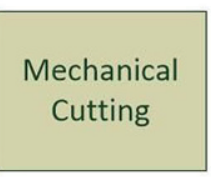

- Pick Tools

- Disk Tools

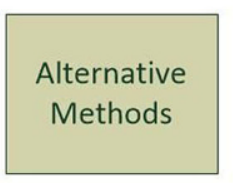

- High pressure water

- Microwaves

- Electropulse

- LASER

- Heat
Combined Methods

- Microwave \& Cutting

- Microblasting \& Cutting

- Electropulse \& Cutting

- High pressure water \& Cutting de die Maschine auch mehrfach adaptiert. Als nächstes soll eine ansteigende $270^{\circ}$ Wendelstrecke aufgefahren werden.

Die bisherigen täglichen Vortriebsleistungen werden mit $4 \mathrm{~m}$ angegeben.

\section{Alternative oder kombinierte/hybride Lösemethoden}

Wo Bohr-und-Sprengtechnik oder mechanisches Schneiden aus verschiedenen Gründen nicht eingesetzt oder an ihre Leistungsgrenzen geraten könnten alternative oder kombinierte/hybride Lösemethoden ein Weiterkommen ermöglichen.

Alle alternativen Gesteinslösemethoden nach Abb. 16 wurden in den vergangenen Jahren weltweit intensiv erforscht, sind jedoch alleine viel zu energieintensiv im Abbau und mit sonstigen Nachteilen behaftet und daher derzeit wirtschaftlich keine Alternative im Hartgesteins-Bergbau.

Für den Vortrieb in Hartgesteinsstrecken könnten jedoch kombinierte/hybride Methoden, bei denen mittels Einsat- zes von Mikrowelle, Microblasting, Elektropulse oder Hockdruckwasser das Gestein „vorgeschädigt“ wird und damit das mechanische Schneiden erleichtern, eine wirtschaftliche Möglichkeit eröffnen. Daher ist in diesem Bereich Grundlagenforschung im Gange [23, 24].

\section{Zusammenfassung}

Während in der Gewinnung großer Erzmengen Bohren und Sprengen die optimale Methode ist, werden im Streckenvortrieb im Hartgestein Schritt für Schritt auch mobile und flexible mechanische Vortriebssysteme verfügbar. Diese neuartigen Systeme sind aber alle noch im Prototypenstadium, und es wird wohl noch ein weiteres Jahrzehnt an Verbesserungen benötigen, bis die gewünschten Vortriebsleistungen erreicht werden.

Für konventionelle Teilschnittmaschinen mit Rundschaftmeißeln war die Verbesserung der Meißeltechnik in Bezug auf Standfestigkeit und erweitertem Einsatzbereich in den letzten 20 Jahren leider nur im einstelligen Prozentbereich. 
Verbunden mit Automation werden diese Rapid Mine Development Systeme im kommenden Jahrzehnt zur Einführung kommen. Wie erfolgreich sie sein werden, hängt auch wesentlich von der Verbesserung der Qualität und Lebensdauer der Schneidwerkzeuge im Hartgestein ab.

Kombinierte/hybride Lösungsmethoden könnten einen wesentlichen Beitrag für die Mechanisierung des Vortriebs im Hartgestein leisten.

Auf jeden Fall ist noch eine Menge Forschungs- und Entwicklungsarbeit zu leisten und es bleibt zu hoffen das die Bergbauindustrie für die notwendigen Investitionen bereit ist.

Funding. Open access funding provided by Montanuniversität Leoben.

Interessenkonflikt. N. A. Sifferlinger gibt an, dass kein Interessenkonflikt besteht.

Open Access Dieser Artikel wird unter der Creative Commons Namensnennung 4.0 International Lizenz veröffentlicht, welche die Nutzung, Vervielfältigung, Bearbeitung, Verbreitung und Wiedergabe in jeglichem Medium und Format erlaubt, sofern Sie den/die ursprünglichen Autor(en) und die Quelle ordnungsgemäß nennen, einen Link zur Creative Commons Lizenz beifügen und angeben, ob Änderungen vorgenommen wurden.

Die in diesem Artikel enthaltenen Bilder und sonstiges Drittmaterial unterliegen ebenfalls der genannten Creative Commons Lizenz, sofern sich aus der Abbildungslegende nichts anderes ergibt. Sofern das betreffende Material nicht unter der genannten Creative Commons Lizenz steht und die betreffende Handlung nicht nach gesetzlichen Vorschriften erlaubt ist, ist für die oben aufgeführten Weiterverwendungen des Materials die Einwilligung des jeweiligen Rechteinhabers einzuholen.

Weitere Details zur Lizenz entnehmen Sie bitte der Lizenzinformation auf http://creativecommons.org/licenses/by/4.0/deed.de.

\section{Literatur}

1. Sifferlinger, N.A., Hartlieb, P., Moser, P.: The importance of research on alternative and hybrid rock extraction methods. Berg Hüttenm. Monatsh. 162(2), 58-66 (2017)

2. Boloz, L., Kalukiewicz, A.: Machines for mechanical mining of hardly workable and abrasive rocks. MAPE 3(1), 150-160 (2020)

3. CAT: Longwall automation. https://www.cat.com/en_US/by-industry/ mining/articles/longwall-automation-download.html, Zugegriffen: 18. Nov. 2020

4. Südwestsalz: Umstieg auf schneidende Gewinnung hat sich für Bergwerk bewährt. https://allgemeinebauzeitung.de/abz/salzberg werk-umstieg-auf-schneidende-gewinnung-hat-sich-fuer-bergwerkbewaehrt-35473.html, Zugegriffen: 27. Dez. 2020

5. Pickering, R., Smit, A., Moxham, K.: Mining by rock cutting in narrow reefs. In: International Platinum Conference 'Platinum Surges Ahead'. The Southern African Institute of Mining and Metallurgy, (2006)

6. Bergbau Newsletter 19. KW: Hard rock Strebtechnik von Caterpillar (2016). https://docplayer.org/107835419-Bergbaunewsletter-19-kwhard-rock-strebtechnik-von-caterpillar.html, Zugegriffen: 28. Dez. 2020
7. Wirtgen: Surface miner. https://www.wirtgen-group.com/ocs/de-at/ wirtgen/surface-miner-74-c/, Zugegriffen: 27. Dez. 2020

8. Restner, U., Plinninger, R.J.: Rock mechanical aspects of roadheader excavation. In: 64th Geomechanical Colloquium EUROCK 15, Salzburg. (2015)

9. International Mining Magazine: Element Six synthetic diamond will help hard rock cutting to achieve true potential (2019). https://immining.com/tag/mx650/, Zugegriffen: 27. Dez. 2020

10. Maidl, B., Schmid, L., Ritz, W., Herrenknecht, M.: Hardrock Tunnel Boring Machines. Ernst \& Sohn, Berlin (2008)

11. Bilgin, N., Copur, H., Balci, C.: Mechanical Excavation in Mining and Civil Industries. Taylor \& Francis, Boca Raton (2014)

12. Herrenknecht: Gripper-TBM Catalogue. https://www.herrenknecht. com/de/produkte/kernprodukte/tunnelling/gripper-tbm.html, Zugegriffen: 2. Sept. 2016

13. Cigla, M., Yagiz, S., Ozdemir, L.: Application of tunnel boring machines in underground mine development. http://inside.mines.edu/ UserFiles/File/earthMechanics/tbm/tbm3.pdf, Zugegriffen: 25. Dez. 2016

14. CIMMagazine: Hard rock revolution on the horizon (2017). https:// magazine.cim.org/en/technology/hard-rock-revolution-on-the-hori zon/, Zugegriffen: 30. Dez. 2020

15. Williams, L.: Aker Wirth's MTM under way at Rio's North Parkes copper/gold mine. http://www.mineweb.com/archive/aker-wirths-mtmunder-way-at-rios-northparkes-coppergold-mine/, Zugegriffen: 2. Sept. 2016

16. Lyly, J., Hartwig, S., Nord, G.: Hardrock cutting now a reality; 25 March 2018; Bergdagarna (2018). http://www.befoonline.org/ UserFiles/Dokument/Bergdagarna_2018/Artiklar_2018/BT_11.pdf, Zugegriffen: 27. Dez. 2020

17. Canadian Mining Journal: EPIROC introduces mechanical miners (2018). https://www.canadianminingjournal.com/features/epirocintroduces-mechanical-miners/, Zugegriffen: 27. Dez. 2020

18. EPIROC: Maschinen für den mechanischen Gesteinsabbau - Mobile Miner. https://www.epiroc.com/de-at/products/mechanical-rockexcavation, Zugegriffen: 1. Jan. 2021

19. EIT Raw Materials: Kava project Rock Vader, Smart hard rock mining system. https://eitrawmaterials.eu/project/rock-vader/, Zugegriffen: 27. Dez. 2020

20. International Mining Magazine: (2020). https://im-mining.com/ 2020/11/16/sandvik-mining-president-says-battery-electric-linewill-soon-include-65-t-underground-truck/, Zugegriffen: 27. Dez. 2020

21. Robbins Company: Robbins develops rectangular rock mining machine for flat invert (2021). https://www.robbinstbm.com/mdm5000/, Zugegriffen: 8. Dez. 2021

22. Robbins Company: MDM 5000. https://www.robbinstbm.com/ products/mining-machines/mine-development-machine/, Zugegriffen: 8. Dez. 2021

23. Hartlieb, P., Grafe, B.: Experimental study on microwave assisted hard rock cutting of granite. BHM 162(2), 77-81 (2017)

24. Hartlieb, P., Rostami, J.: Rock mechanics implications of microwave treatment of rock as part of a hybrid system for mechanical excavation of rock for civil and mining applications. In: Proceedings of the 2018 European Rock Mechanics Symposium EUROCK. (2018). Chapter 10, 7 Seiten

Hinweis des Verlags. Der Verlag bleibt in Hinblick auf geografische Zuordnungen und Gebietsbezeichnungen in veröffentlichten Karten und Institutsadressen neutral. 